\title{
Application of Multi-sensor Information Fusion Based on Improved Particle Swarm Optimization in Unmanned System Path Planning
}

\author{
https://doi.org/10.3991/ijoe.v13i08.7199 \\ Guolong Shi, Yigang He $\left.{ }^{\varpi}\right)$, Bing Li, Qiwu Luo \\ Hefei University of Technology, Hefei, P.R. China. \\ $694698336 @$ qq. com
}

\begin{abstract}
Intelligent vehicle driving performance is safe and stable, which can significantly improve the efficiency of road traffic and reduce energy consumption, and intelligent vehicle is also the development direction of modern transport. Its core technology is intelligent environment perception module, by using a variety of sensors on the car in which the surrounding environment for data collection, processing module to provide effective control for the basis. In this paper, a new SINS / CNS / GPS integrated navigation observation equation is proposed, and a new federated data fusion structure is designed for the integrated navigation system. The particle filter is used to fuse the multi-source data of the federated filter subsystem, thus eliminating the limitations of the classical Kalman filter. The traditional Kalman filter structure and the federal particle filter mechanism are designed. The comparison shows that the proposed algorithm is effective in the information fusion of the integrated navigation system, and the filtering effect is superior to the traditional filtering method.
\end{abstract}

Keywords_-particle filter; information fusion; intelligent vehicle; navigation

\section{Introduction}

With the progress of society, the car has become an essential travel means of transport, vehicle congestion, traffic accidents and other issues are increasingly apparent. The rapid growth in the number of cars is caused by the low efficiency of public transport which leads to frequent traffic accidents. The establishment of a modern intelligent transportation system will be mentioned on the agenda. Intelligent Vehicles, as an important part of Intelligent Transportation Systems, is also the main body of the system, which not only improves driving safety and road traffic efficiency, but also reduces energy consumption [1-3]. Due to many advantages, the technology research has been increasingly concerned about the relevant institutions at home and abroad. Intelligent transportation system can effectively relieve traffic pressure, rational allocation of public transport resources and road resources. Based on machine sensing technology and control technology, the driving system uses information transmission technology and computer vision technology helps to monitor the road 
environment, road signs, traffic signs, other vehicles, pedestrians and traffic accidents, so as to ensure the safe driving of intelligent vehicles in various road conditions [4]. And it can be some of the abnormal conditions for timely processing. In the past 10 years, the relevant technology has made great progress, and some countries have successfully developed a number of vision-based road identification or tracking system. Among them, representative systems include LOIS system, GOLD system, RALPH system, SCARF system and ALVINN system. From the application of these advanced technology can be seen, perception of external environment module is the core technology of intelligent vehicles [5]. Each navigation system has its own performance and scope of application have some limitations; it is difficult to fully meet the unmanned navigation requirements, in order to improve the navigation system accuracy and reliability requirements by using two methods. 1) To improve the accuracy of the device itself. 2) The best combination of navigation devices. The most commonly used vehicle navigation system is inertial navigation system (INS), global positioning system (GPS), astronomical navigation system (CNS), taking into account the advantages and disadvantages of the three navigation methods, there are a lot of literature which will be a combination of the three to study.

At present, Kalman filter is the most widely used multi-sensor information fusion technology which is the general use of distributed or federated filter structure. However, the classical Kalman filter algorithm used by the data processing of the federated filtering subsystem does not achieve the desired effect when dealing with nonGaussian, nonlinear states and parameter estimates, since the Kalman filter is used to obtain the optimal estimation result. 1) The statistical characteristics of the external jamming noise are subject to the Gaussian distribution. 2) The statistical characteristics of the external jamming noise are subject to the Gaussian distribution [6, 7]. Almost all systems in the real world have non-linear, non-Gaussian features, it is difficult to meet the classic Kalman filter use conditions. Extended Kalman filter is usually used when the state equation or the measurement equation is nonlinear. EKF applies the Taylor expansion of the nonlinear function to the first order linearization, ignoring the other higher order terms, so that the nonlinear problem is transformed into linear, and the Kalman linear filtering algorithm can be applied to the nonlinear system. This solves the problem of system nonlinearity. EKF has been widely used by non-linear state estimation systems and has been widely used by people. However, this method also brings two shortcomings [8-10]. One is that when strong nonlinearity, $E K F$ violates local linear assumption, Taylor expansion $E K F$ algorithm may make the filter divergence. In addition, because $E K F$ linearization process requires Jacobian matrix, its cumbersome calculation process leads to the realization of the method which is relative to the $E K F$ algorithm. Therefore, $E K F$ is the suboptimal filter under the minimum variance criterion.

The recently studied particle filter algorithm uses a series of weighted random particles sampling to approximate the posterior probability density function, which is a Bayesian estimation method based on Monte Carlo [11]. Therefore, it is independent of the system model, The limitation of linearization or Gaussian noise is not only possible to solve the error which is caused by linearization of EKF, but also it can avoid errors which is caused by $U K F$ due to non-Gaussian $P D F$ and measurement 
model in any environment. In this paper, a new type of fault-tolerant information fusion algorithm is named federated particle filter, which can deal with non-Gaussian and nonlinear systems is adopted [12]. The algorithm uses particle filtering to deal with the multi-source data of the federated filtering subsystem.

\section{Material and methods}

\subsection{Establishment of Integrated Navigation Model}

In order to accurately plan the navigation path of the unmanned system, the state of the system needs to be accurately described [13]. Here, the state variable is selected as the attitude error, the velocity error and the position error of the platform. The state equation is as follow.

$$
\dot{X}(t)=F(t) X(t)+G(t) w(t)
$$

And $\boldsymbol{X}(\mathrm{t})=\left[\begin{array}{lllllllll}\phi_{E} & \phi_{N} & \phi_{U} & \delta v_{E} & \delta v_{N} & \delta v_{U} & \delta L & \delta \lambda & \delta h\end{array}\right]^{\mathrm{T}}$ is used as state variables, $\mathrm{E}, \mathrm{N}, \mathrm{U}$ represent geographic coordinate system in the east, north, day three directions, $\phi_{E}, \phi_{N}, \phi_{U}$ is used for the platform error angle, $\delta v_{E}, \delta v_{N}, \delta v_{U}$ means the speed error, $\delta L, \delta \lambda, \delta h$ represents position error.

The equations (2) to (4) are the attitude error equations of the platform.

$$
\begin{aligned}
\dot{\phi}_{E}= & \phi_{N}\left(\omega_{i e} \sin L+\frac{v_{E} \tan L}{R_{N}+h}\right)-\phi_{U}\left(\omega_{i e} \cos L+\frac{v_{E}}{R_{N}+h}\right)-\frac{\delta v_{N}}{R_{M}+h}+\delta h \frac{v_{N}}{\left(R_{N}+h\right)^{2}}- \\
& C_{11}\left(\varepsilon_{b x}+\varepsilon_{r x}+\omega_{g x}\right)-C_{21}\left(\varepsilon_{b y}+\varepsilon_{r y}+\omega_{g y}\right)-C_{31}\left(\varepsilon_{b z}+\varepsilon_{r z}+\omega_{g z}\right) \\
\dot{\phi}_{N}= & -\phi_{E}\left(\omega_{i e} \sin L+\frac{v_{E} \tan L}{R_{N}+h}\right)-\phi_{U}\left(\frac{v_{N}}{R_{N}+h}\right)-\delta L \omega_{i e} \sin L+\frac{\delta v_{E}}{R_{N}+h}+\delta h \frac{v_{E}}{\left(R_{N}+h\right)^{2}}- \\
& C_{12}\left(\varepsilon_{b x}+\varepsilon_{r x}+\omega_{g x}\right)-C_{22}\left(\varepsilon_{b y}+\varepsilon_{r y}+\omega_{g y}\right)-C_{32}\left(\varepsilon_{b z}+\varepsilon_{r z}+\omega_{g z}\right) \\
\dot{\phi}_{U}= & \phi_{E}\left(\omega_{i e} \cos L+\frac{v_{E}}{R_{N}+h}\right)+\phi_{N}\left(\frac{v_{N}}{R_{M}+h}\right)+\delta L\left(\omega_{i e} \cos L+\frac{v_{E} \sec ^{2} L}{R_{N}+h}\right)+\frac{\delta v_{E} \tan L}{R_{N}+h}- \\
& \frac{\delta h v_{E} \tan L}{\left(R_{N}+h\right)^{2}}-C_{13}\left(\varepsilon_{b x}+\varepsilon_{r x}+\omega_{g x}\right)-C_{23}\left(\varepsilon_{b y}+\varepsilon_{r y}+\omega_{g y}\right)-C_{33}\left(\varepsilon_{b z}+\varepsilon_{r z}+\omega_{g z}\right)
\end{aligned}
$$

And $\phi_{E} 、 \phi_{\mathrm{N}} \& \phi_{\mathrm{U}}$ respectively, IMU system mathematical platform along the east, north, day to the attitude error angle, $\delta v_{E} 、 \delta v_{N}$ and $\delta v_{U}$ is the carrier of the east, north, days to the speed error, $\delta L, \delta \lambda, \delta h$ is the carrier geography error.

The equations $(5) \sim(7)$ for the system speed error equation is shown as follow. 


$$
\begin{aligned}
& \delta \dot{v}_{E}=-\phi_{N} f_{U}+\phi_{U} f_{N}+\delta v_{E} \frac{v_{N} \tan L-v_{U}}{R_{N}+h}+\delta v_{N}\left(2 \omega_{i e} \sin L+\frac{v_{E} \tan L}{R_{N}+h}\right)- \\
& \delta v_{U}\left(2 \omega_{i e} \cos L+\frac{v_{E}}{R_{N}+h}\right)+\delta L\left[2 \omega_{i e}\left(v_{U} \sin L+v_{N} \cos L\right)+\frac{v_{E} v_{N} \sec ^{2} L}{R_{N}+h}\right] \\
& +\delta h \frac{v_{E} v_{U}-v_{E} v_{N} \tan L}{\left(R_{N}+h\right)^{2}}+C_{11}\left(\nabla_{b x}+\omega_{a x}\right)+C_{21}\left(\nabla_{b y}+\omega_{a y}\right)+C_{31}\left(\nabla_{b z}+\omega_{a z}\right) \\
& \delta \dot{v}_{N}=-\phi_{U} f_{E}+\phi_{E} f_{U}-2 \delta v_{E}\left(\omega_{i e} \sin L+\frac{v_{E} \tan L}{R_{N}+h}\right)-\delta v_{N} \frac{v_{U}}{R_{M}+h}-\delta v_{U} \frac{v_{N}}{R_{M}+h} \\
& -\delta L\left(2 v_{E} \omega_{i e} \cos L+\frac{v_{E}^{2} \sec ^{2} L}{R_{N}+h}\right)+\delta h\left[\frac{v_{N} v_{U}}{\left(R_{M}+h\right)^{2}}+\frac{v_{E} \tan L}{\left(R_{N}+h\right)^{2}}\right]+ \\
& C_{12}\left(\nabla_{b x}+\omega_{a x}\right)+C_{22}\left(\nabla_{b y}+\omega_{a y}\right)+C_{32}\left(\nabla_{b z}+\omega_{a z}\right) \\
& \delta \dot{v}_{U}=\phi_{N} f_{E}-\phi_{E} f_{N}+2 \delta v_{E}\left(\omega_{i e} \cos L+\frac{v_{E}}{R_{N}+h}\right)+\delta v_{N} \frac{2 v_{N}}{R_{M}+h}-2 \delta L v_{E} \omega_{i e} \sin L- \\
& \delta h\left[\frac{v_{E}}{\left(R_{N}+h\right)^{2}}+\frac{v_{N}}{\left(R_{M}+h\right)^{2}}\right]+C_{13}\left(\nabla_{b x}+\omega_{a x}\right)+C_{23}\left(\nabla_{b y}+\omega_{a y}\right)+C_{33}\left(\nabla_{b z}+\omega_{a z}\right)
\end{aligned}
$$

In the equations (5) to (7), the components, $f_{E}, f_{N}$ and $f_{U}$ are in the east, north, and earth directions, and $\nabla_{b x} 、 \nabla_{b y}$ and $\nabla_{b z}$ means the accelerometer on the carrier axis drifts randomly $[14,15]$. The acceleration $\omega_{a x}, \omega_{a y}, \omega_{a z}$ is the acceleration on the carrier $\mathrm{x}, \mathrm{y}$ and $\mathrm{z}$ White noise.

The equations (8) to (10) are the system position error equations.

$$
\begin{gathered}
\delta \dot{L}=\delta v_{N} \frac{1}{R_{M}+h}-\delta h \frac{v_{N}}{\left(R_{M}+h\right)^{2}} \\
\delta \dot{\lambda}=\delta v_{E} \frac{\sec L}{R_{N}+h}+\delta L \frac{v_{E} \tan L \sec L}{R_{N}+h}-\delta h \frac{v_{E} \sec L}{\left(R_{N}+h\right)^{2}} \\
\delta \dot{h}=\delta v_{U}
\end{gathered}
$$

In the formulas (8) to (10), the latitude error is $\delta L, \delta \lambda$ is a longitude error and $\delta h$ is used as a height error [16]. 
The equation (1) is discredited to the given formula (11)

$$
\boldsymbol{X}_{k}=\boldsymbol{\Phi}_{k, k-1} \boldsymbol{X}_{k-1}+\boldsymbol{\Gamma}_{k-1} \boldsymbol{W}_{k-1}
$$

$\boldsymbol{X}_{k}$ is n-D State vector at time $\mathrm{K}, \boldsymbol{\Phi}_{k, k-1}$ is step-state transition matrix $(\mathrm{N} \times \mathrm{n}$ order $)$ between time k-1 to time $\mathrm{k}, \boldsymbol{W}_{k-1}$ is the system noise (r dimension) at time k-1, $\boldsymbol{\Gamma}_{k-1}$ is the system noise matrix ( $N \times r$ order).

\subsection{Integrated Navigation Model Measurement Equation}

INS / CNS / GPS integrated navigation system has two sets of observations, namely INS / CNS combined navigation observation and INS / GPS integrated navigation observation.

1) INS / CNS integrated navigation algorithm, through the navigation solution, INS outputs attitude quaternion $\hat{Q}_{n}^{b}$. Star sensor provides the corresponding attitude quaternion.

$$
\widehat{Q}_{b}^{n}=Q_{b}^{I} \otimes Q_{I}^{i} \otimes Q_{i}^{e} \otimes\left(Q_{e}^{n} \otimes Q_{n}^{n^{n}}\right) \otimes Q_{n^{\prime}}^{n} \otimes\left(\left[1, \frac{v}{2}\right]^{\mathrm{T}}\right)
$$

$\otimes$ means the quaternion product operation, $\mathrm{n}$ is the navigation coordinate system, $n^{\prime}, n^{\prime \prime}$ indicates the actual geographic coordinate system.

The attitude quaternary of the carrier of the star sensor $Q_{b}^{I}$ is relative to the equatorial inertial coordinate system. The attitude quaternion of the equatorial inertial coordinate system $Q_{I}^{i}$ relative to the inertia coordinate system of the launching point, the position of the inertial coordinate system of the launching point relative to the earth coordinate system $Q_{i}^{e}$ is determined by the geostationary angular rate stored by the navigation computer. The position of the earth coordinate system relative to the navigation coordinate system $Q_{e}^{n}$ is determined by the position information of the output $[17,18]$. The flexural error quasi-quaternion $Q_{n}^{n^{n}}$ between the earth coordinate system and the navigation coordinate system caused by the positioning error of strap down inertial navigation, the attitude quaternion $Q_{n^{\prime}}^{n}$ corresponding to the error angle error of the mathematical platform of INS, Small.

$$
Q_{n^{\prime}}^{n}=\left[1,-\frac{\varphi_{E}}{2},-\frac{\varphi_{N}}{2},-\frac{\varphi_{U}}{2}\right]^{\mathrm{T}}
$$


$\left[1, \frac{v}{2}\right]^{\mathrm{T}}$ is used for the star sensor observation error corresponding to the attitude quaternion.

Based on the above parameters can be obtained measurement equation.

$$
\begin{aligned}
& Z_{1}=-C_{b}^{n} \varphi+C_{b}^{n}\left[\begin{array}{ccc}
-1 & 0 & 0 \\
0 & \cos L & 0 \\
0 & \sin L & 0
\end{array}\right]\left[\begin{array}{c}
\delta L \\
\delta \lambda \\
\delta h
\end{array}\right]+v_{C}=H_{C} X+v_{C}
\end{aligned}
$$

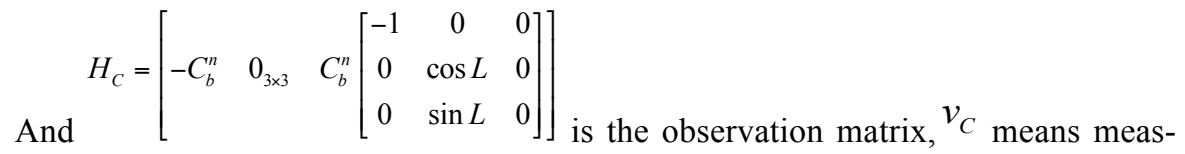
uring noise.

2) In the INS / GPS integrated navigation algorithm, we select the speed and position given by the inertial navigation system, and the velocity and position difference given by the GPS are measured.

$$
Z^{\prime}=\left[\begin{array}{c}
v_{I E}-v_{G E} \\
v_{I N}-v_{G N} \\
\left(L_{I}-L_{G}\right) R_{M} \\
\left(\lambda_{I}-\lambda_{G}\right) R_{N} \cos L
\end{array}\right]=\left[\begin{array}{c}
\delta v_{E} \\
\delta v_{N} \\
R_{M} \delta L \\
R_{N} \cos L \delta \lambda
\end{array}\right]+\left[\begin{array}{c}
v_{E} \\
v_{N} \\
N_{E} \\
N_{N}
\end{array}\right]
$$

$v_{E}, v_{N}$ the GPS receiver along the east and north direction of the speed error, $N_{E}, N_{N}$ the GPS receiver along the east and north direction of the position

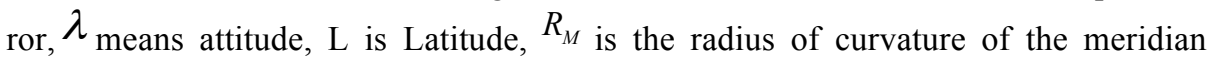
circle, $R_{N}$ is the radius of curvature of the system.

According to $Z^{\prime}$, we can obtain INS / GPS measurement equation.

$$
Z_{2}=H_{G} X(k)+v_{G}
$$

And $H_{G}=\left[\begin{array}{ll}0_{4 \times 3} & \operatorname{diag}\left(1,1, R_{M}, R_{N} \cos L\right)_{4 \times 4} \quad 0_{4 \times 2}\end{array}\right]$ is the observation matrix, $v_{G}$ is the measurement noise.

\subsection{Combined positioning path planning}

In order to compare the filtering effect with the common Kalman filter, this paper designs the Kalman filter structure and the structure of the federal particle filter.

Kalman filter. Kalman filter basic equation gives state one - step prediction equation. 


$$
\hat{\boldsymbol{X}}_{k, k-1}=\boldsymbol{\Phi}_{k, k-1} \hat{\boldsymbol{X}}_{k-1}
$$

We can get one step prediction of mean square error equation.

$$
\boldsymbol{P}_{k, k-1}=\boldsymbol{\Phi}_{k, k-1} \boldsymbol{P}_{k-1} \boldsymbol{\Phi}_{k, k-1}^{\mathrm{T}}+\boldsymbol{\Gamma}_{k, k-1} \boldsymbol{Q}_{k-1} \boldsymbol{\Gamma}_{k, k-1}^{\mathrm{T}}
$$

Filter gain equation is here.

$$
\boldsymbol{K}_{k}=\boldsymbol{P}_{k, k-1} \boldsymbol{H}_{k}^{\mathrm{T}}\left[\boldsymbol{H}_{k} \boldsymbol{P}_{k, k-1} \boldsymbol{H}_{k}^{\mathrm{T}}+\boldsymbol{R}_{k}\right]^{-1}
$$

State estimation equation is expressed as follows.

$$
\hat{\boldsymbol{X}}_{k}=\hat{\boldsymbol{X}}_{k, k-1}+\boldsymbol{K}_{k}\left[\boldsymbol{Z}_{k}-\boldsymbol{H}_{k} \hat{\boldsymbol{X}}_{k, k-1}\right]
$$

Estimation of Mean Square Error Equation is given here.

$$
\boldsymbol{P}_{k}=\left[\boldsymbol{I}-\boldsymbol{K}_{k} \boldsymbol{H}_{k}\right] \boldsymbol{P}_{k, k-1}
$$

The equations (17) to (21) are the basic equations of Kalman filter for stochastic linear discrete systems.

Figure 1 describes the above discrete Kalman filter algorithm, we can see from the figure Kalman filter which has two calculation loops [19, 20]. Among them, the gain calculation circuit is an independent calculation circuit, and the filter calculation circuit depends on the gain calculation circuit.

INS/CNS/GPS integrated navigation system block diagram is shown in Figure 2.

Path planning of federated particle swarm optimization algorithm. Particle Swarm Optimization is a non-parametric Monte Carlo simulation method which helps to implement recursive Bayesian filtering. It can also be applied to any nonlinear system which can be described by the state space model. The precision can be approximated to the optimal estimate. Figure 3 is a schematic diagram of a particle swarm filter algorithm that describes the three steps of time update, observation update, and re-sampling contained in a particle swarm filter algorithm. The prior probability of time is approximated by the particle with the weight value. During the time ${ }^{k-1}$ updating process, the state of each particle $N$ is predicted by the system state transition equation $x_{k-1}^{(i)}$. After observing the value, update the particle weights is $\tilde{w}_{k}^{(i)}$. The resampling process discards smaller particles and replaces them with larger weights, and the weights of the particles are reset to $1 / N$. 


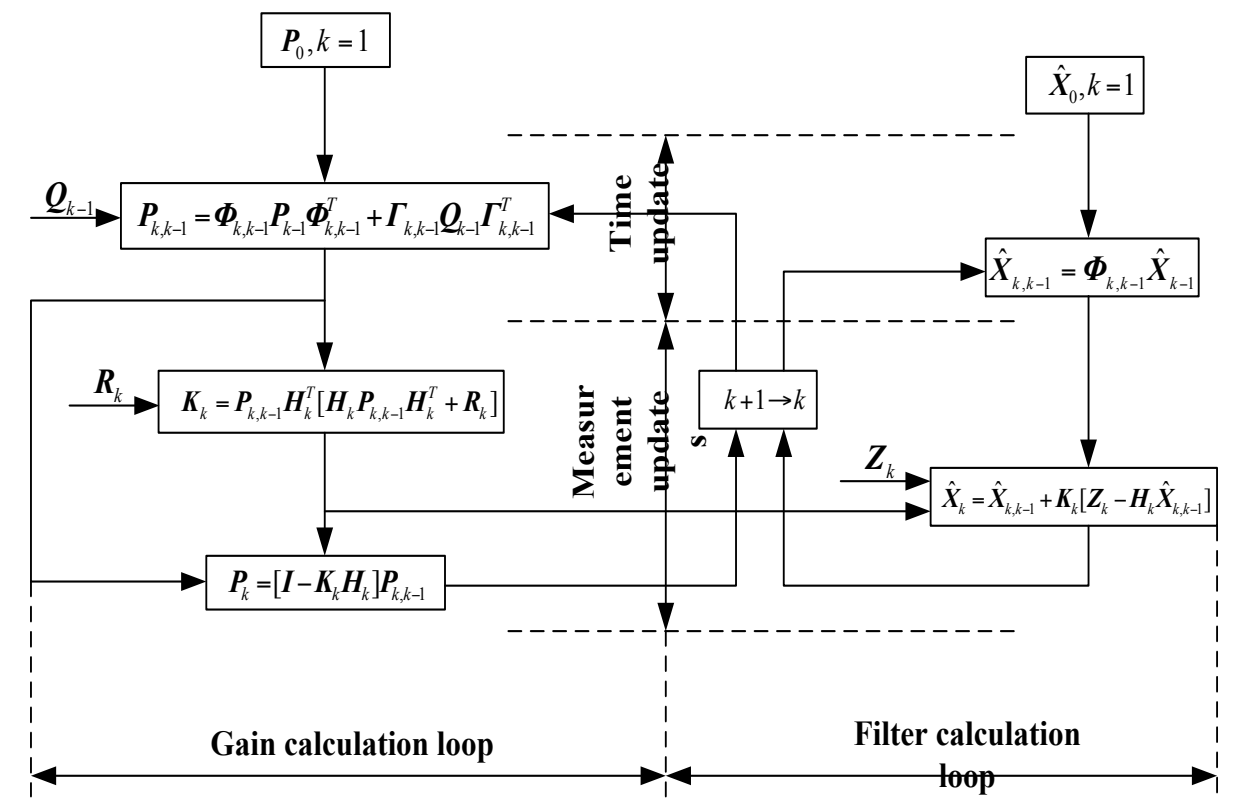

Fig. 1. Flow chart of discrete Kalman filter algorithm

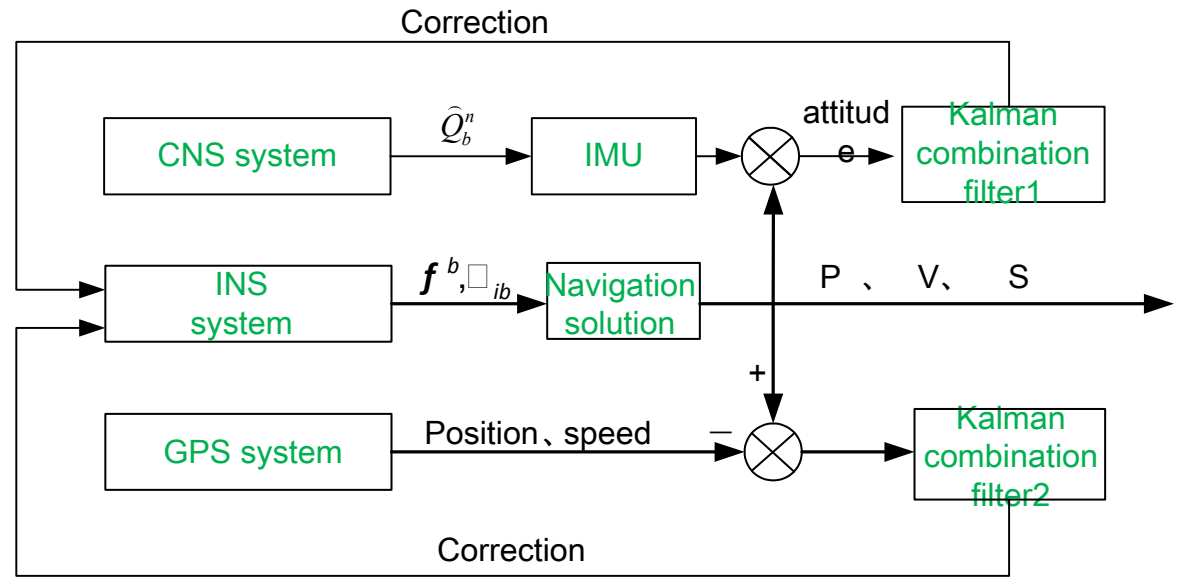

Fig. 2. INS / CNS / GPS integrated navigation system Kalman filter structure 


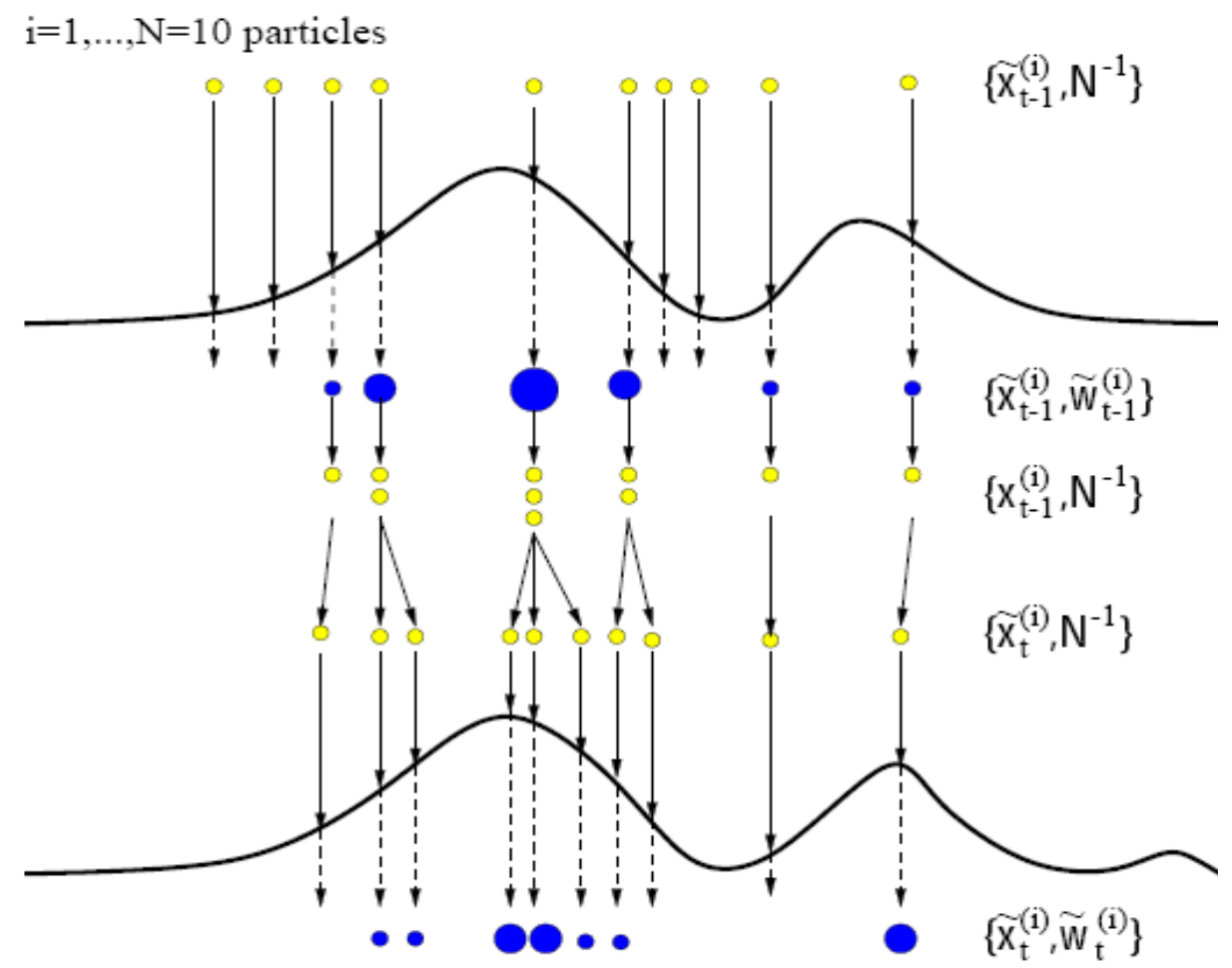

Fig. 3. Schematic diagram of particle filter group algorithm

Standard flow of particle swarm filter algorithm is show as follows.

(1) Particle swarm initialization is $k=0$.

For $i=1,2, \mathrm{~L}, N$, the sample $\left\{x_{0}^{(i)}\right\}_{i=1}^{N}$ is generated by a priori sampling $p\left(x_{0}\right)$.

(2) For $k=1,2, \mathrm{~L}$, the loop performs the following steps.

(3) Importance sampling. For $i=1,2, \mathrm{~L}, N$, the importance of probability density generated in the sampling of particles $\left\{\tilde{x}_{k}^{(i)}\right\}_{i=1}^{N}$, calculate the weight of the particle $\tilde{w}_{k}^{(i)}$, and normalization;

(4) Re-sampling. The particle group $\left\{\tilde{x}_{k}^{(i)}, \tilde{w}_{k}^{(i)}\right\}$ is used to re-sampling after the particle group $\left\{x_{k}^{(i)}, 1 / N\right\}$.

(5) Output. Calculate the time estimate of the time is $\hat{x}_{k}=\sum_{i=1}^{N} \tilde{x}_{k}^{(i)} \tilde{w}_{k}^{(i)}$.

The problem of weight degradation in particle swarm optimization is unavoidable. Although the re-sampling method can alleviate the weight degradation phenomenon to a certain extent, the re-sampling method can also cause some other problems. Resampling needs to be integrated with all the particles to achieve, limiting the parallel computation of particle swarm optimization. In addition, according to the principle of 
re-sampling, the larger weight of the particles will be more of the selected copy [21]. After a number of iterations, it will inevitably lead to the same particles more and more, the lack of diversity of particles, particle degradation may occur, So that the state estimation produces a large deviation. In order to solve the problem of highdimensional non-Gaussian, nonlinear system's state and parameter estimation, particle filter is introduced into the federated filter, and the federated particle filter algorithm is obtained [22]. Its structure is shown in Figure 4.

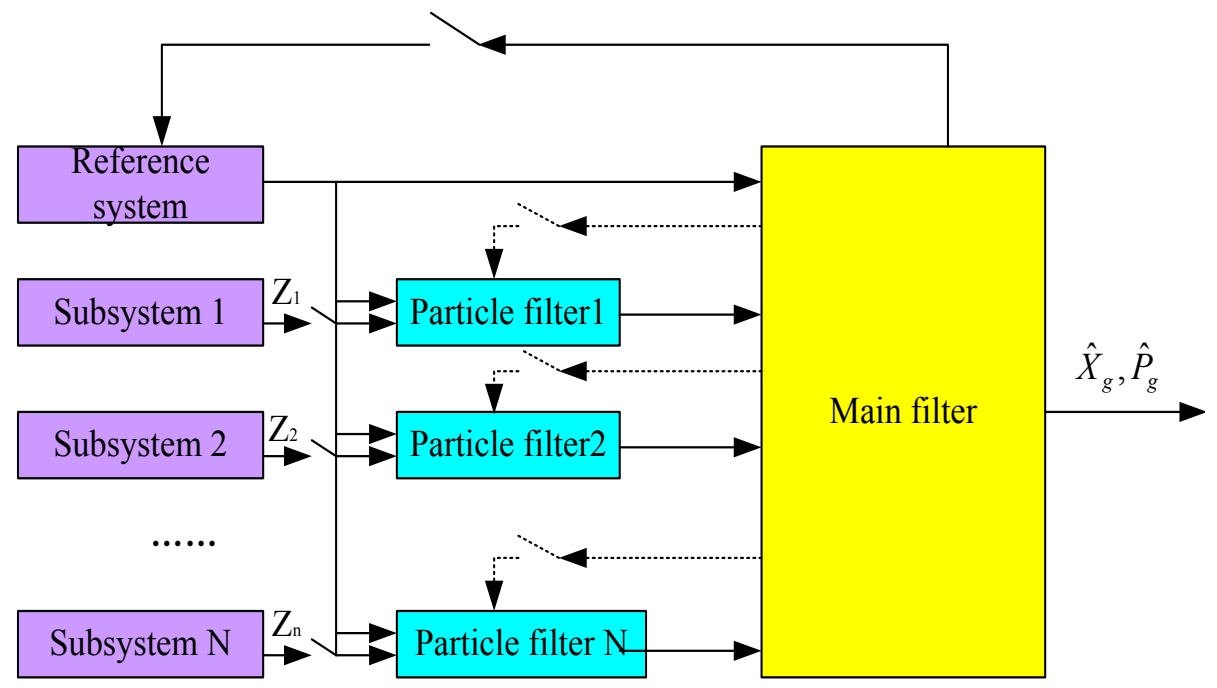

Fig. 4. Federal particle swarm filter structure

Figure 4 in the public reference system, its output on the one hand directly to the main filter, on the other hand output to the sub-filter as a measurement value. The sub-filter is used to calculate the n-group local optimal estimate $\hat{X}_{i}, \hat{P}_{i}(i=1,2, \mathrm{~L} n)$ of the system by the particle filter according to the state equation and the measurement equation. Then, the n-group local optimal estimate $\hat{X}_{g}, \hat{P}_{g}$ is sent to the main filter for global optimal information fusion. Finally, after each filtering phase is completed, the global filters will feedback the results $\hat{X}_{g}, \hat{P}_{g}$ to each sub-filter and the main filter according to the principle of "information conservation".

\subsection{Particle fitness function}

In this paper, based on the path length as the fitness function, the safety and smoothness are added, and all the parameters are weighted average to meet the requirements of unmanned system path planning in complex environment.

1) The planned path is as short as possible and the length of the path can be expressed by follows. 


$$
f_{1}=\sum_{i=1}^{d} \sqrt{\left(x_{i+1}-x_{i}\right)^{2}+\left(\mathrm{y}_{i+1}-y_{i}\right)^{2}}
$$

And $f_{1}$ represents the sum of the straight-line distances between all adjacent vertices in a particle, $\left(x_{i}, y_{i}\right)$ represents the current coordinates of the particle, and $\left(x_{i+1}, y_{i+1}\right)$ is representing the coordinates of the particle next position.

2) The introduction of the penalty functions helps to improve the safety of the path. When the robot collides with the obstacle, a penalty function is introduced on the path length of the unmanned system. The more the obstacle is, the greater the penalty is imposed, and the smaller the probability of the path is generated. The penalty function is expressed as follows.

$$
f_{2}=\sum_{k=1}^{N} M
$$

Where $\mathrm{N}$ is the sum of the number of obstacles on the straight path from the starting point to the end point, and $\mathrm{M}$ represents a given constant term.

3) Introduce path smoothness. Unmanned system in the course of the process of walking diagonal route can shorten the length of the path to save time, but change the direction of motion of unmanned systems which will be time-consuming, in order to make the system to complete the task of the shortest time; this paper introduces the path smoothness Formula.

$$
f_{3}=\frac{n_{1} \pi r}{4}+\frac{n_{2} \pi r}{2}
$$

$n_{1}$ means the number of times the robot which is rotated by $45^{\circ}$ in the diagonal path of the particle, ${ }_{2}$ is expressed as the number of times the robot which is rotated by $90^{\circ}$ in the right-angled path, and $\mathrm{r}$ is the radius of the robot. The comprehensive fitness function is $f_{1}, f_{2}, f_{3}$.

$$
F=\alpha \cdot f_{1}+\beta \cdot f_{2}+\gamma \cdot f_{3}
$$

In the formula, the weighting factor for each function is $\alpha, \beta, \gamma$ of which any real number greater than or equal to zero. By adjusting $\alpha, \beta, \gamma$, you can adjust $f_{1} 、 f_{2} 、 f_{3}$ by the proportion of the fitness function. When $\alpha=1, \beta=0, \gamma=0$, it is converted to a conventional mathematical model with only the path length as a fitness function [23]. In the formula $f_{2}$ and $f_{3}$ as a penalty judgment function and 
smoothness correction function, the value is generally less than the order of magnitude.

$$
F=\alpha \cdot f_{1}+\beta \cdot f_{2}+\gamma \cdot f_{3}
$$

\section{Discussions and results}

\subsection{Discussions}

In a complex road environment, a single sensor has its limitations. We just install a single sensor which is difficult to provide a comprehensive description of the road environment, so the design of intelligent vehicles must be equipped with a variety of sensors. Such as the night when the infrared sensor is essential, radar detection of the surrounding obstacles near the machine vision in addition to daily applications and other sensors can be combined with the intelligent vehicle driving safety which can be more reliable. With the development of computer information technology, communication technology, control technology and electronic technology, the application of multi-sensor information fusion technology in intelligent vehicle technology research has made many exciting achievements, such as automotive systems interconnection technology, the European Peugeot system, the US IVHS system. Tsai-Hong Hong and other use of laser sensors to collect images to obtain the distance information in front of the vehicle, in the normal road environment, the use of color cameras and laser sensors to jointly understand the road surface and locate the road boundary. These technologies have been continually improved and are believed to have revolutionized the automotive industry in the near future.

On the two-dimensional plane, the working environment of the unmanned system is modeled by the improved raster method. The effectiveness of environmental modeling is critical to the efficient planning and obstacle avoidance of unmanned systems. The advantages of raster methods are the ease of modeling and the ability to use multiple grids to represent multiple obstacles. Reducing the damage caused by obstacle modeling in the feasible area is necessary. In order to ensure that the unmanned system can move without collision in the environmental model, the model is simplified to a small particle size, and the actual size of the unmanned system is converted into the area of the obstacle. In this paper, the boundary of the obstacle is expanded outward according to the actual size of the unmanned system. If there is an obstacle in a grid, the black raster is defined as a barrier grid, which is expressed as 1; otherwise, the white is a free raster, which is expressed as 0 . The use of multiple grids to represent different shapes of obstacles, the free grid is merged into the reach of the unmanned system area.

In this paper, when the space is modeled, the obstacle in the space is treated as follows. The actual obstacle is shown in Figure 5 (a), and the obstruction after treatment is shown in Figure 5 (b). 


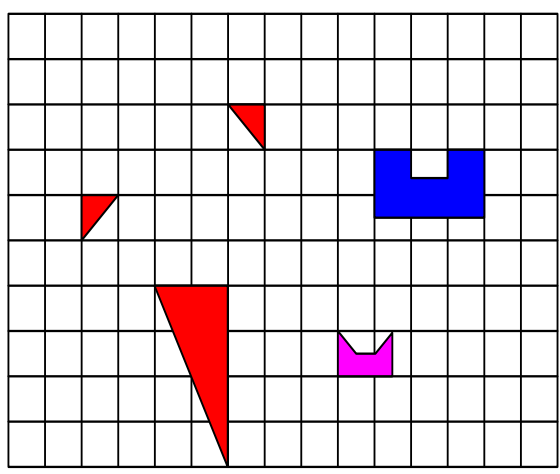

(a) The shape of the obstacle after treatment

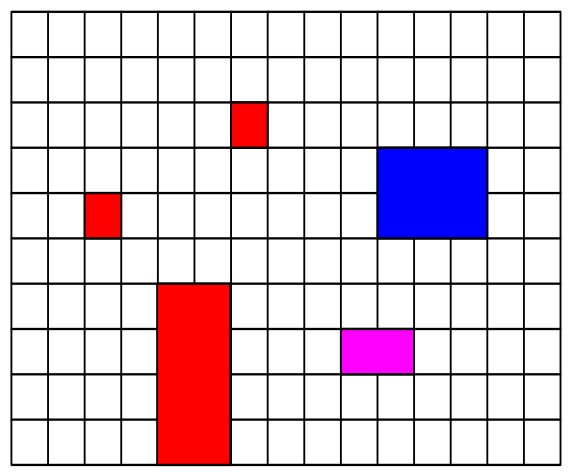

(b) The shape of the obstruction after treatment

Fig. 5. Obstacle shape treatment

1. When a grid is less than a grid.

2. The empty part of the obstacle and the obstacle is shown as a whole obstacle, to avoid the emergence of local dead zone.

3. Treat the border of the map as an obstacle.

In the established spatial model, the particle swarm optimization algorithm is used to find an optimal path. Each particle in the particle swarm algorithm has a solution, that is, a path, particle swarm algorithm from a large number of solutions is used to find an optimal solution and form the optimal path.

When planning a path, it is necessary to determine the best path based on the effective particle to determine the effectiveness of the particle. The validity of a particle means that the rectangular area between any two adjacent elements in the particle can be freely connected and there is no obstacle in the middle. That is, the particles satisfying the constraints and passing the grids as free grids are valid particles, as long as any two adjacent elements in the particle are not free to connect or the path in the same row (same column) in the grid. The particles are invalid. Figure 6 is an effective path in the path planning process.
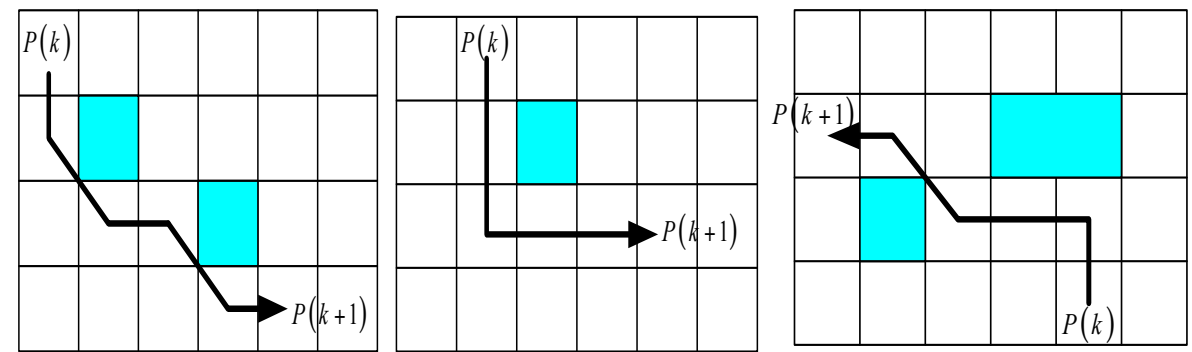

Fig. 6. Effective path graph 


\subsection{Results and analysis}

In this paper, the simulation software Matlab is used to simulate the computer. The simulation parameters are as follows, population size is the number of particles $\mathrm{M}=$ 100 , the maximum particle velocity $v_{\max }=20$, the maximum number $k_{\max }=500 \mathrm{of}$ iterations is the acceleration factor $c_{1}=c_{2}=4$ of algorithm, the maximum inertia weight is $w_{\max }=0.85$, the minimum inertia weight is $w_{\min }=0.3$, and the fixed inertia weight is $w=0.75$.

The Kalman filter algorithm and the federated particle swarm algorithm are simulated by using the grid model. The simulation results in the complex obstacle environment model are shown in Figure 7 and Figure 8.

From the simulation results can be seen, the unmanned system from the starting point "S" to the end of the "T", the two algorithms can achieve the ability to find and avoid obstacles. However, Kalman filter algorithm unmanned system path planning is easy to fall into the local optimal, too much in the obstacle avoidance to consider the impact of obstacles, while ignoring the real-time, and the federal particle swarm filter unmanned system path planning can jump out of the local best. In this paper, considering the influence of the edge of the obstacle, the safety and smoothness of the path length are introduced, and the simulation is 80 times. The experimental record is shown in Table 1.

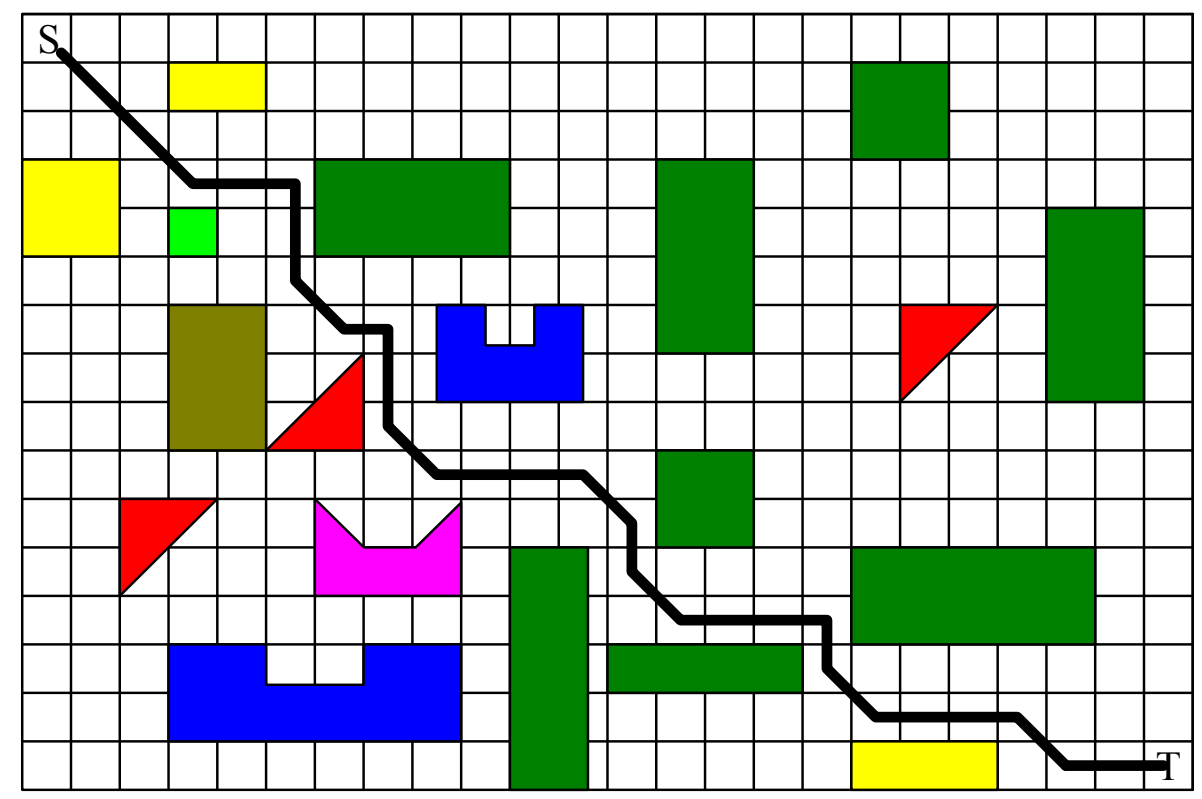

Fig. 7. Kalman filter algorithm in complex environment Unmanned system path planning 


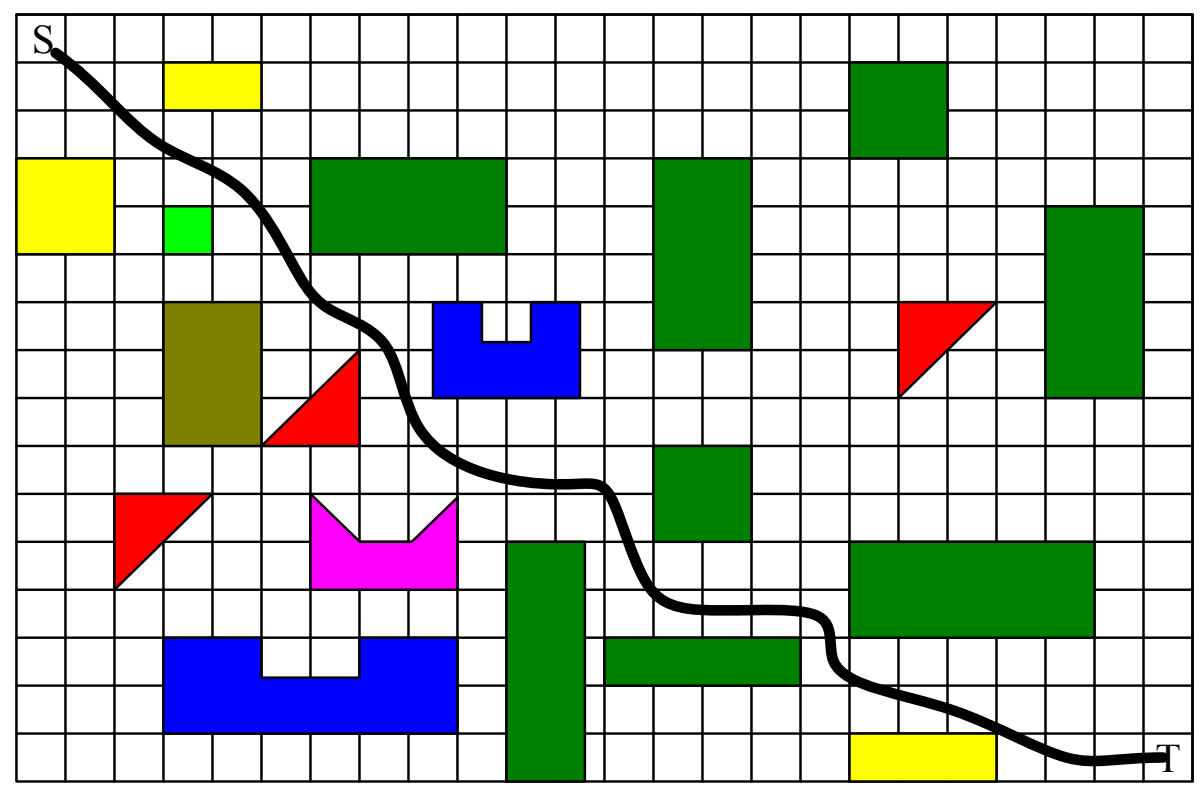

Fig. 8. Agile Particle Swarm Optimization Algorithm for Unmanned Systems in Complex Environment

Table 1. Comparison of simulation results

\begin{tabular}{|l|c|c|c|c|c|}
\hline \multirow{2}{*}{ Algorithm } & \multicolumn{3}{|c|}{ Path length } & \multicolumn{2}{c|}{ Simulation time } \\
\cline { 2 - 6 } & Optimal path & Mean & Variance & Mean & Variance \\
\hline Kalman filter algorithm & 35.71 & 42.39 & 128.514 & 0.28 & $4.87 \mathrm{e}-3$ \\
\hline $\begin{array}{l}\text { Federal Particle Swarm } \\
\text { Optimization }\end{array}$ & 25.36 & 33.43 & 113.067 & 0.21 & $2.52 \mathrm{e}-3$ \\
\hline
\end{tabular}

It can be seen from Table 1 that the optimal path of the federated particle swarm algorithm is the shortest. Due to the introduction of the fitness function, the convergence speed of the federated particle swarm algorithm is larger than that of the Kalman filter in the robot path planning application, and the optimal path is shorter and the running time is also shorter. The federated particle swarm algorithm is superior to the Kalman filter algorithm regardless of the optimal path length or running time.

\section{Conclusions}

In the intelligent vehicle environment perception module technology research, the sensor is the key of intelligent vehicle control system. How to make the sensor technology better applied to the automotive industry up, the future will become a sensor technology research in the field of development. The integration of various types of sensor technology to provide intelligent vehicles with more reliable and reliable road environment information, the development of intelligent automotive technology is 
essential. Due to the actual application of the information obtained by the environment is mostly uncertain information, sensor feedback information fusion to restore the real road conditions there are still great difficulties.

Multi-sensor information fusion Federal particle swarm filter as the realization of unmanned system path planning is based on parallel filtering technology and information sharing principle, so it has the advantages of good real-time, fault-tolerant and high precision. In this paper, a highly potential nonlinear filtering algorithm which is a particle swarm filter is introduced into the federated filtering structure, and it extends the federated filtering to solve the system state, parameter estimation and path planning in nonlinear complex environments. In the field, computer simulation proves that this method can not only find the optimal path, but also the algorithm is easy to implement and it can get better results than the traditional Kalman filter algorithm.

\section{$5 \quad$ Acknowledgment}

This work was supported by the National Natural Science Foundation of China under Grant No. 51577046, the State Key Program of National Natural Science Foundation of China under Grant No. 51637004, the National Key Research and Development Plan "Important Scientific Instruments and Equipment Development" Grant No. 2016YFF0102200.

\section{References}

[1] Cappello, F., Sabatini, R., Ramasamy, S., \& Marino, M. (2015, June). Particle filter based multi-sensor data fusion techniques for RPAS navigation and guidance. In Metrology for Aerospace (MetroAeroSpace), 2015 IEEE (pp. 395-400). IEEE. https://doi.org/10.1109/ MetroAeroSpace.2015.7180689

[2] Cappello, F., Ramasamy, S., Sabatini, R., \& Liu, J. (2015, June). Low-cost sensors based multi-sensor data fusion techniques for RPAS navigation and guidance. In Unmanned Aircraft Systems (ICUAS), 2015 International Conference on (pp. 714-722). IEEE. https://doi.org/10.1109/ICUAS.2015.7152354

[3] Pajares, G. (2015). Overview and current status of remote sensing applications based on unmanned aerial vehicles (UAVs). Photogrammetric Engineering \& Remote Sensing, 81(4), 281-329. https://doi.org/10.14358/PERS.81.4.281

[4] ZHANG, Pin, W. H. Dong, and D. D. Gao. "AN optimal method of data fusion for multisensors based on Bayesian estimation." Chinese Journal of Sensors and Actuators 27.5 (2014): 643-648.

[5] Li, Q., Chen, L., Li, M., Shaw, S. L., \& Nuchter, A. (2014). A sensor-fusion drivableregion and lane-detection system for autonomous vehicle navigation in challenging road scenarios. IEEE Transactions on Vehicular Technology, 63(2), 540-555. https://doi.org/10.1109/TVT.2013.2281199

[6] Blasch, E., Dezert, J., \& Pannetier, B. (2013, May). Overview of Dempster-Shafer and belief function tracking methods. In SPIE Defense, Security, and Sensing (pp. 874506874506). International Society for Optics and Photonics.

[7] Zhang, Z., Li, Y., Wang, F., Meng, G., Salman, W., Saleem, L. \& Liu, Y. (2016). A Novel Multi-Sensor Environmental Perception Method Using Low-Rank Representation and a 
Paper-Application of Multi-sensor Information Fusion Based on Improved Particle Swarm Optimiza...

Particle Filter for Vehicle Reversing Safety.Sensors, 16(6), 848. https://doi.org/10.3390/ $\underline{\text { s16060848 }}$

[8] Yassin, A., Nasser, Y., Awad, M., Al-Dubai, A., Liu, R., Yuen, C. \& Aboutanios, E. (2016). Recent advances in indoor localization: A survey on theoretical approaches and applications. IEEE Communications Surveys \& Tutorials.

[9] Sabatini, R., Ramasamy, S., Cappello, F., \& Gardi, A. (2014, December). RPAS navigation and guidance systems based on GNSS and other low-cost sensors. In proceedings of 4th Australasian Conference on Unmanned Systems (ACUS 2014), Melbourne, Australia.

[10] Gao, Shesheng, Gaoge $\mathrm{Hu}$, and Yongmin Zhong. "Windowing and random weighting $\square$ based adaptive unscented Kalman filter." International Journal of Adaptive Control and Signal Processing 29.2 (2015): 201-223. https://doi.org/10.1002/acs.2467

[11] Zhang, Y., Chao, A., Zhao, B., Liu, H., \& Zhao, X. (2016, August). Migratory birdsinspired navigation system for unmanned aerial vehicles. In Information and Automation (ICIA), 2016 IEEE International Conference on (pp. 276-281). IEEE. https://doi.org/10.1109/ICInfA.2016.7831835

[12] Salavasidis, G., Harris, C., McPhail, S., Phillips, A. B., \& Rogers, E. (2016, November). Terrain Aided Navigation for Long Range AUV operations at arctic latitudes. In Autonomous Underwater Vehicles (AUV), 2016 IEEE/OES (pp. 115-123). IEEE. https://doi.org/10.1109/AUV.2016.7778658

[13] Echeverri, A. F., Medeiros, H., Walsh, R., Reznichenko, Y., \& Povinelli, R. (2017). Hierarchical Bayesian Data Fusion for Robotic Platform Navigation.arXiv preprint arXiv:1704.06718.

[14] Zhang, Y. M., Wang, W., Sui, J., \& Wang, X. Y. (2014). A GPS-Assisted Calibration Algorithm for Misalignment Angle between Star Sensor and Strap-Down Inertial Navigation System. In Applied Mechanics and Materials(Vol. 635, pp. 1457-1463). Trans Tech Publications.

[15] Santoso, Fendy, Matthew A. Garratt, and Sreenatha G. Anavatti. "Visual-Inertial Navigation Systems for Aerial Robotics: Sensor Fusion and Technology." IEEE Transactions on Automation Science and Engineering14.1 (2017): 260-275. https://doi.org/10.1109/ TASE.2016.2582752

[16] Tsai, G. J., Chiang, K. W., Chu, C. H., Chen, Y. L., El-Sheimy, N., \& Habib, A. (2015). The Performance Analysis of AN Indoor Mobile Mapping System with Rgb-D Sensor. The International Archives of Photogrammetry, Remote Sensing and Spatial Information Sciences, 40(1), 183. https://doi.org/10.5194/isprsarchives-XL-1-W4-183-2015

[17] Li H, Ding W, Cao X, et al. Image Registration and Fusion of Visible and Infrared Integrated Camera for Medium-Altitude Unmanned Aerial Vehicle Remote Sensing[J]. Remote Sensing, 2017, 9(5): 441. https://doi.org/10.3390/rs9050441

[18] Donavanik, D., Hardt-Stremayr, A., Gremillion, G., Weiss, S., \& Nothwang, W. (2016, May). Multi-sensor fusion techniques for state estimation of micro air vehicles. In SPIE Defense+ Security (pp. 98361V-98361V). International Society for Optics and Photonics.

[19] Cappello, Francesco, Subramanian Ramasamy, and Roberto Sabatini. "A low-cost and high performance navigation system for small RPAS applications." Aerospace Science and Technology 58 (2016): 529-545. https://doi.org/10.1016/j.ast.2016.09.002

[20] Ferreira, F., Machado, D., Ferri, G., Dugelay, S., \& Potter, J. (2016, September). Underwater optical and acoustic imaging: A time for fusion? a brief overview of the state-of-the-art. In OCEANS 2016 MTS/IEEE Monterey(pp. 1-6). IEEE.

[21] Kealy, A., Retscher, G., Toth, C., Hasnur-Rabiain, A., Gikas, V., Grejner-Brzezinska, D., ... \& Moore, T. (2015). Collaborative navigation as a solution for PNT applications in 
Paper-Application of Multi-sensor Information Fusion Based on Improved Particle Swarm Optimiza..

GNSS challenged environments-report on field trials of a joint FIG/IAG working group. Journal of Applied Geodesy, 9(4), 244-263. https://doi.org/10.1515/jag-2015-0014

[22] Serranoa, D., de Haag, M. U., Dill, E., Vilardaga, S., \& Duan, P. (2014). Seamless indooroutdoor navigation for unmanned multi-sensor aerial platforms. The International Archives of Photogrammetry, Remote Sensing and Spatial Information Sciences, 40(3), 115. https://doi.org/10.5194/isprsarchives-XL-3-W1-115-2014

[23] Zhu, H., Yuen, K. V., Mihaylova, L., \& Leung, H. (2017). Overview of Environment Perception for Intelligent Vehicles. IEEE Transactions on Intelligent Transportation Systems. https://doi.org/10.1109/TITS.2017.2658662

[24] Cucci, D. A., Migliavacca, M., Bonarini, A., \& Matteucci, M. (2016). Development of mobile robots using off-the-shelf open-source hardware and software components for motion and pose tracking. In Intelligent Autonomous Systems 13 (pp. 1451-1465). Springer International Publishing. https://doi.org/10.1007/978-3-319-08338-4 104

\section{$7 \quad$ Authors}

Guolong Shi, Yigang He (Corresponding Author), Bing Li, and Qiwu Luo are with School of Electrical Engineering and Automation, Hefei University of Technology, Hefei, 230009, P.R. China (694698336@qq.com).

Article submitted 25 May 2017. Published as resubmitted by the authors 29 june 2017. 